\title{
PREGUNTAS Y PROCESOS COGNITIVOS EN TEXTOS ESCOLARES CHILENOS DE HISTORIA, GEOGRAFÍA Y CIENCIAS SOCIALES
}

\author{
Questions and Cognitive Processes in Chilean School Textbooks \\ of History, Geography and Social Sciences
}

\author{
Andrea Minte Münzenmayer ${ }^{1}$ \\ ORCID: 0000-0002-8720-692X \\ Alejandro Sepúlveda Obreque ${ }^{2}$ \\ ORCID: 0000-0002-5033-8400 \\ Danilo Díaz-Levicoy ${ }^{3}$ \\ ORCID: 0000-0001-8371-7899 \\ Denis Igor Obando ${ }^{4}$ \\ ORCID: 0000-0003-2349-3816 \\ 12 Universidad de Los Lagos, Chile. \\ ${ }^{3}$ Universidad Católica del Maule, Chile. \\ ${ }^{4}$ Departamento de Administración de Educación Municipal de Puerto Montt, Chile. \\ Correos: andrea.minte@ulagos.cl; asepulve@ulagos.cl; dddiaz01@ hotmail.com; denis.igor@dempuertomontt.cl
}

Recibido: 25/09/2020

Aceptado: 08/04/2021

Resumen: La presente investigación tiene como objetivo determinar la dimensión del proceso cognitivo que promueven las preguntas contenidas en los textos escolares de Historia, Geografía y Ciencias Sociales de la educación básica en Chile. Se trató de un estudio cuantitativo en el cual se analizaron 1775 preguntas a la luz de la taxonomía de Anderson y Krathwohl (2001). Se aplicaron pruebas estadísticas no paramétricas a los datos obtenidos. Entre los resultados se evidencia que los textos escolares presentan preguntas de nivel memorístico y reproductivo, lo cual incide en un bajo desarrollo cognitivo de los estudiantes. Las preguntas que demandan niveles cognitivos superiores representan un mínimo porcentaje, a pesar de la importancia que revisten para el desarrollo de competencias intelectuales, específicamente, de pensamiento histórico.

Palabras clave: preguntas escritas; procesos cognitivos; textos escolares; educación básica; Chile.

Abstract: The present study aims to determine the dimension of the cognitive process promoted by the questions contained in the school textbooks of History, Geography and Social Sciences of basic education in Chile. It was a quantitative study in which 1775 questions were analyzed considering the taxonomy of Anderson and Krathwohl (2001). Non-parametric statistical tests were applied to the data obtained. Among the results, it is evident that the school texts present questions of memoristic and reproductive level, which leads to a low cognitive development of the students. Questions that demand higher cognitive levels represent a minimum percentage, despite their importance for the development of intellectual competencies, specifically, historical thinking.

Keywords: written questions; cognitive process; school textbooks; basic education; Chile. 


\section{Introducción}

La formulación de preguntas no es tarea banal. Requiere de la experticia de los profesores, ya sea que se formulen de manera oral o escrita. Lo mismo ocurre con las preguntas contenidas en los textos de Historia, Geografía y Ciencias Sociales de la enseñanza básica chilena, objeto de interés en esta investigación. En investigaciones relacionadas con preguntas, la mayoría refiere a textos narrativos que han evaluado el aprendizaje memorístico de hechos mediante preguntas (Boudreau, Wood, Willoghby y Specht, 1999; Pressley, Symons, McDaniel, Snyder y Turner, 1988; Woloshyn, Wood, Silloughby y Pressley, 1990), lo cual solo potencia la pasividad de los estudiantes (Wood, 1988).

Las preguntas relacionadas con textos escritos y también las contenidas en las propuestas de actividades didácticas en los textos escolares, en general, tienen la función de dirigir la atención de los estudiantes hacia aspectos importantes de la información. Ello incide en la representación mental que se crea con el texto (Van den Broek, Tzeng, Risden, Trabasso y Basche, 2001).

Friedman y Rickards (1981) señalan que la comprensión es mayor al insertar preguntas inferenciales y de parafraseo que las demandas de las preguntas de tipo textual, las cuales requieren o esperan una respuesta literal del texto. Adicionalmente, este tipo de preguntas mejora las respuestas a ítems textuales, inferenciales y de parafraseo. Esto da origen a lo que ellos denominaron: efecto de transferencia de nivel. Los autores concluyen que las preguntas adjuntas a textos permiten mejorar el nivel cognitivo y de procesamiento de la información por parte de los lectores.

En cuanto a la complejidad cognitiva requerida en los textos escolares, Bel y Colomer (2018) distinguen tres niveles en las actividades didácticas de los textos escolares. El nivel 1 representa complejidad cognitiva baja; el nivel 2, complejidad media, y el nivel 3 complejidad cognitiva alta. Este último nivel implica "la resolución de preguntas inferenciales y la aplicación de contenidos procedimentales como estrategias" (Bel y Colomer, 2018, p. 13).

En el análisis de las actividades didácticas propuestas en los textos escolares chilenos de Historia, Sáez-Rosenkranz (2017, p. 37) señala que la "estructuración se presenta de forma inversa a las recomendaciones para la secuenciación de contenidos...y en el caso de los objetivos de aprendizaje (Anderson y Krathwohl, 2001), los cuales por norma general deben ser progresivos". 
Esta situación conlleva una adquisición parcial de aprendizajes relacionados con el pensamiento histórico, lo cual se encuentra en un $60 \%$ de las actividades didácticas pesquisadas en textos escolares de $5 .^{\circ}$ y $6 .^{\circ}$ año de enseñanza básica en Chile.

Por otra parte, Sáiz y Colomer (2014) sostienen que los niños pueden aprender historia y desarrollar el pensamiento histórico a temprana edad, a diferencia de los postulados piagetanos que establecen lo contrario. La empatía y la contextualización se aprenden desde la infancia. Los procesos cognitivos propios del pensamiento histórico pueden ser aprendidos por niños de corta edad y, de forma progresiva, desarrollar habilidades de pensamiento histórico. Se puede fomentar el cultivo de competencias cognitivas altas mediante los textos escolares y, específicamente, a partir de las lecturas, las actividades didácticas y las preguntas.

Wimer, Ridenour, Thomas y Place (2001) destacan que las preguntas de alto nivel cognitivo suscitan el análisis, la síntesis, la evaluación, de tal forma que establecen las bases para el desarrollo de habilidades de pensamiento crítico; en cambio, las preguntas que demandan bajo nivel de respuestas solo logran el recuerdo y la memorización. Las preguntas de alto nivel, llamadas también elaborativas (Ozgungor y Guthrie, 2004) son aquellas que activan procesos cognitivos complejos. Entre sus funciones se encuentran: 1) dirigir la atención hacia aspectos destacados de un texto; 2) fomentar conexiones entre el conocimiento previo del lector y la información contenida en el texto; 3) monitorear la comprensión (Ozgungor y Guthrie, 2004).

Otros estudios (McDaniel y Donnelly, 1996; Ozgungor y Guthrie, 2004; Seifert, 1993) han evaluado el efecto de las preguntas elaborativas y la generación de inferencias. También se ha investigado acerca de la relación entre este tipo de preguntas y las representaciones mentales. Se ha concluido que los efectos son positivos y las representaciones mentales son más coherentes, tanto en estudiantes de enseñanza básica (Wood, Pressley y Winne, 1990) como en estudiantes universitarios (Ozgungor y Guthrie, 2004; Pressley, McDaniel, Turnure, Wood y Ahmad, 1987).

Las denominadas preguntas de pensamiento crítico son aquellas que inducen procesos cognitivos de alto nivel, tales como el análisis de ideas, la comparación-contraste, las inferencias, las predicciones y las evaluaciones, entre otros (King, 1995). En situaciones de comprensión de textos, los lectores tratan de generar diferentes tipos de inferencias: asociativas, explicativas y predictivas (Trabasso y Magliano, 1996) y, de acuerdo con estas, se han clasificado las preguntas en tres categorías, a saber: preguntas asociativas (con formulaciones interrogativas (quién, qué, cómo, cuándo, dónde); preguntas explicativas (por qué); y preguntas predictivas (qué pasaría después, qué pasaría si...). 
De acuerdo con Carretero y Montanero (2008) existen dos habilidades fundamentales para desarrollar capacidades de pensamiento histórico en los estudiantes. Estas son: la capacidad de comprensión del tiempo histórico y, la de valoración e interpretación crítica de fuentes. "Pensar históricamente supone... mucho más que acumular información sobre hechos sobresalientes del pasado" (Carretero y Montanero, 2008, p. 136). Es decir, pensar históricamente implica una exigencia cognitiva compleja que en los textos escolares se promueven mediante los textos escritos, las imágenes, las actividades didácticas y las preguntas formuladas en estas.

Las preguntas que apuntan al desarrollo del pensamiento crítico también son denominadas abiertas o de aplicación, debido a que invitan a dar respuestas tentativas, exploratorias y creativas. Las respuestas requieren aplicar los conocimientos previos a nuevas situaciones. Las preguntas de alto nivel evidencian aprendizajes profundos, comprensión y creatividad (André, 1990).

Por otra parte, Gallástegui y Otero (2008) propusieron cinco dimensiones que definen la calidad de una pregunta: 1) tipo de entidad a que está referida (objetos o procesos); 2) tipo de inferencia que el sujeto trata de realizar; 3) proximidad de la entidad que afecta la pregunta (medio próximo o lejano); 4) nivel de concreción de las entidades (concretas o abstractas); 5) transgresión o no de una expectativa clara.

Graesser, McMahen y Johnson (1994) reflexionan acerca de la clase de preguntas que conviene formular a los estudiantes con la finalidad de estimular el aprendizaje y concluyen que son aquellas que: 1) solventan déficits de conocimiento; 2) examinan y comprueban el conocimiento común; 3) coordinan acciones sociales; 4) controlan la conversación y la atención. Sostienen que el primer tipo de preguntas son las más importantes. Otros autores las denominan prototípicas, sinceras o genuinas (Berlyne y Frommer, 1966; Flammer, 1981; Otero y Graesser, 2001; Ram, 1991; Van der Meij, 1994).

Para Torres et al. (2012, p. 50) “en el sistema educativo están mucho más desarrollados los métodos para valorar la calidad de las respuestas que los métodos que valoran la calidad de las preguntas”. Dillon (1990) estudió la generación de preguntas por parte de los estudiantes. Concluyó que el primer paso para la formulación de preguntas es lo que denomina estado de perplejidad. Este se produciría cuando el estudiante, quien maneja conocimientos previos, intenta comprender nueva información, pero esta no se acomoda o integra lógicamente con la preexistente. La incongruencia o sorpresa produce una conciencia de incomprensión, que llevaría a la formulación de una pregunta. Es decir, cuando no se logra cuadrar la nueva información con la 
aprendida antes, surge una pregunta. Y, de acuerdo con Graesser y Olde (2003), La France (1992) o Van der Meij (1990), el conocimiento previo de los estudiantes es clave en la formulación de preguntas. En la medida en que la información previa que posee el sujeto es más amplia se produce un conflicto cognitivo o incomprensión más profunda.

En síntesis, la formulación de preguntas en los textos escolares está directamente asociada a esta idea: cuanto mayor contenido dominen los estudiantes (ya sea que estén en el texto o en otros) y se enuncien preguntas de alto nivel cognitivo, que promuevan la comprensión, el análisis, la síntesis, la aplicación y la creación, surgirán preguntas que tenderán a un aprendizaje de mayor calidad. Por este motivo, es relevante poner atención a las preguntas, que acompañan documentos, ilustraciones o actividades presentes en los textos escolares. En palabras de Gavelek y Raphael (1985, p. 114): "la formulación de preguntas representa uno de los primeros medios mediante el cual los individuos son capaces de avanzar en su propia comprensión y, como tal, representa una poderosa actividad metacognitiva".

El objetivo general de la investigación fue determinar la dimensión del proceso cognitivo que promueven las preguntas contenidas en los textos escolares de Historia, Geografía y Ciencias Sociales de la educación básica chilena. Como objetivos específicos se buscó establecer la relación entre la dimensión del proceso cognitivo, los cursos y las unidades temáticas de los textos.

\section{Método}

Se trató de una investigación cuantitativa, de tipo descriptiva, en la cual se trabajó con los textos escolares de Historia, Geografía y Ciencias Sociales correspondiente a $5 .^{\circ}, 6 .^{\circ}, 7 .^{\circ}$ y $8 .^{\circ}$ año de educación básica, en uso durante 2018. En el Cuadro 1 se presentan los textos analizados.

\section{Cuadro 1}

Textos escolares analizados

Fernández, C. y Giadrosic, G. (2018a). Historia, Geografía y Ciencias Sociales 5. año. SM.

Fernández. C. y Giadrosic, G. (2018b). Historia, Geografía y Ciencias Sociales 6. ${ }^{\circ}$ año. SM

Landa, L. y Pinto, V. (2018a). Historia, Geografía y Ciencias Sociales 7. ${ }^{\circ}$ año. SM

Landa, L. y Pinto, V. (2018b). Historia, Geografía y Ciencias Sociales . $^{\circ}$ año. SM

Fuente: Elaboración propia (2020) 
Los criterios de selección fueron: 1) de reciente publicación; 2) se utilizan actualmente en el nivel de educación básica; 3 ) poseen la mayor cobertura geográfica, dado que el Estado chileno entrega textos escolares al 90\% de la población escolar; 4) su distribución es gratuita y masiva.

Se estudió el total de preguntas formuladas en cada una de las unidades temáticas de los cuatro textos escolares de la investigación correspondientes a los cuatro niveles de educación básica chilena. Se analizaron 1775 preguntas, sin contabilizar aquellas derivadas. Las preguntas se desglosan por curso en la Tabla 1.

Tabla 1

Cantidad de preguntas declaradas por unidades temáticas

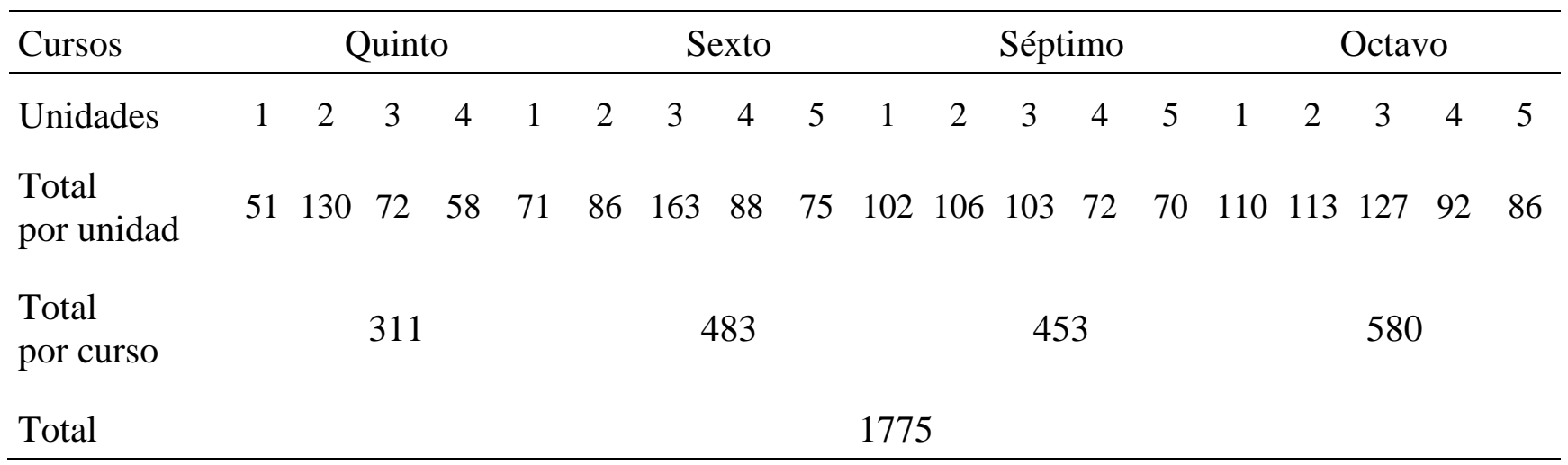

Fuente: Elaboración propia (2020)

Las preguntas se analizaron y categorizaron a partir de Anderson y Krathwohl (2001), quienes proponen una taxonomía para procesos de enseñanza-aprendizaje y evaluación, diseñada en dos dimensiones: la del conocimiento y la de categorías del conocimiento. Esta última plantea la existencia de seis niveles: recordar, comprender, aplicar, analizar, evaluar y crear. Como una forma de operacionalizar cada una de las seis categorías, los investigadores asignan a cada una un listado de habilidades declarados como formas verbales, las cuales permiten especificar la acción intelectual requerida en el proceso de un aprendizaje específico. A partir de lo anterior y, con la necesidad de recabar los datos, se construyó una matriz de datos para consignar el nivel cognitivo de las preguntas de los textos escolares. Con el requerimiento de estimar la validez de contenido del instrumento, mediante el juicio de expertos, se utilizó el modelo de Escobar-Pérez y CuervoMartínez (2008). De esta forma, se ordenaron las preguntas de acuerdo con el nivel taxonómico dado por los verbos previamente identificados. 
El análisis estadístico realizado sobre el proceso cognitivo de las preguntas que se formulan en los textos de estudio de Historia, Geografía y Ciencias Sociales y el curso (respectivamente unidad) se hizo mediante el software SPSS y fue el siguiente:

1) Se inició con un análisis exploratorio del proceso cognitivo de las preguntas (contempla las categorías: recordar, comprender, aplicar, analizar, evaluar y crear), según el curso (posteriormente unidad temática) en el que se plantean, con la finalidad de estudiar el comportamiento porcentual y detectar la presencia o ausencia de diferencias significativas como primera aproximación.

2) Con la finalidad de estudiar la existencia de diferencias estadísticamente significativas en el proceso cognitivo de las preguntas formuladas en los textos escolares, según el curso (posteriormente unidad temática) en el que se plantean, se selecciona la prueba no paramétrica H de Kruskal-Wallis.

Respecto a la aplicación de la prueba $H$ de Kruskall-Wallis en ambos casos estudiados se consideraron los siguientes supuestos:

i) presencia de $\mathrm{K}$ muestras independientes $(\mathrm{K}>2)$.

ii) variable dependiente (proceso cognitivo) de naturaleza ordinal.

iii) variable independiente que genera los grupos (curso y unidad) de naturaleza politómica. En este caso, la variable independiente puede ser politómica nominal o politómica ordinal. (Berlanga Silvente, V. y Rubio Hurtado, M.J. (2012).

3) Finalmente, se examina la existencia de asociación entre las variables proceso cognitivo y curso, así como proceso cognitivo y unidad.

3.1. Proceso cognitivo y curso: se determinó si a medida que aumenta el nivel taxonómico de las preguntas formuladas, ocurría lo propio en el curso. Para estos efectos, se utilizó Rho de Spearman: El supuesto empleado fue que ambas variables estudiadas son categóricasordinales.

3.2. Proceso cognitivo y unidad: se determinó si el nivel taxonómico de las preguntas guardaba alguna relación o dependencia con la unidad en la que se formulaban. Para ello se aplicó Chi cuadrado, ya que las variables que intervinieron fueron categórica-ordinal y categórica-nominal. 


\section{Resultados}

Se presentan los resultados encontrados por dimensión del proceso cognitivo al cual corresponden las preguntas formuladas en los textos escolares, específicamente, por curso y por unidad temática.

\section{Dimensiones del proceso cognitivo desde la formulación de preguntas}

En la Tabla 2 se observa que las preguntas registradas en los textos escolares se agrupan, fundamentalmente, en la dimensión cognitiva recordar, correspondiente al 43,3\%, el cual comprende memorizar datos y fechas, definir conceptos.

La dimensión cognitiva comprensión, que incluye ejemplificar, identificar y justificar ideas fundamentales en un mensaje, corresponde al 22,9\% de las preguntas.

Se advierte en la dimensión aplicar un porcentaje de preguntas equivalente al 7,5\%, como también un 12,8 \% del tipo analizar. Las dimensiones cognitivas evaluar y crear 9,9\% y 3,5\%, respectivamente.

\section{Dimensión del proceso cognitivo de las preguntas y cursos}

La dimensión del proceso cognitivo de las preguntas de los textos por curso, de $5 .^{\circ}$ a $8 .^{\circ}$ año básico se presenta en la Tabla 2. Se observa en todos ellos que la dimensión recordar posee el más alto porcentaje de preguntas. Los datos fluctúan entre 53,1 \% y 36,4 \%. Ejemplo de ello es la pregunta:

¿Qué región de Chile conoces? (Texto escolar de Historia, Geografía y Ciencias Sociales, $8 .^{\circ}$ básico, p. 235).

Se evidencia que la dimensión comprensión agrupa la segunda mayoría de preguntas en los textos analizados. En todos los cursos el porcentaje se encuentra entre $25 \%$ y $21,5 \%$. Un ejemplo es la pregunta:

¿Qué significa que los conquistadores y luego los colonizadores se convirtieran en indianos? (Texto escolar de Historia, Geografía y Ciencias Sociales, 5. básico, p. 177).

La dimensión aplicar, es escasamente promovida mediante las preguntas en los textos escolares. En todos los cursos el porcentaje no supera el 10,2 \%. Ejemplo de ello es la siguiente pregunta: 
¿Qué aspectos de la vida actual provienen del Neolítico? (Texto escolar de Historia, Geografía y Ciencias Sociales, 7. ${ }^{\circ}$ básico, p. 39).

Se constata que las preguntas de dimensión cognitiva analizar, están presentes entre un $8,4 \%$ y $18,6 \%$ en los textos, con mayor presencia en los cursos séptimo y octavo año. Ejemplo de este tipo de pregunta:

¿Por qué un principio como el bien común es parte de la constitución? (Texto escolar de Historia, Geografía y Ciencias Sociales, $6 .^{\circ}$ básico, p. 27 ).

Situación similar, en términos de bajo porcentaje, se observa en la categoría evaluar. Los guarismos fluctúan entre 5,1\% y 7,2 \% en quinto y sexto y, 14,1\% y 11,6 \% en séptimo y octavo. Pregunta de esta categoría es:

¿Qué fortalezas y debilidades crees que tienes para participar en una agrupación ciudadana? (Texto escolar de Historia, Geografía y Ciencias Sociales, $6 .^{\circ}$ básico, p. 37).

Hay coincidencia también en el porcentaje de preguntas que promueve la dimensión crear. Es la menos promovida, el rango va de $3,2 \%$ en quinto a $2,8 \%$ en octavo; pasando por $2,9 \%$ en sexto y 5,3\% en séptimo curso. Ejemplo de pregunta correspondiente a esta categoría es la siguiente:

¿Cuáles son los principales riesgos naturales que afectan a la localidad en que vives y qué medidas es necesario tomar para prevenirlos? (Texto escolar de Historia, Geografía y Ciencias Sociales, 6. ${ }^{\circ}$ básico, p. 59).

\section{Tabla 2}

Frecuencia (y porcentaje) de la dimensión del proceso cognitivo de las preguntas por curso

\begin{tabular}{|c|c|c|c|c|c|}
\hline Proceso cognitivo & $5 .^{\circ}$ & $6 .^{\circ}$ & $7 .^{\circ}$ & $8 .^{\circ}$ & Total \\
\hline Recordar & $165(53,1)$ & $245(50,7)$ & $167(36,9)$ & $192(36,4)$ & $769(43,3)$ \\
\hline Comprender & $73(23,5)$ & $104(21,5)$ & $98(21,6)$ & $132(25)$ & $407(22,9)$ \\
\hline Aplicar & $21(6,8)$ & $36(7,5)$ & $46(10,2)$ & $30(5,7)$ & $133(7,5)$ \\
\hline Analizar & $26(8,4)$ & $49(10,1)$ & $54(11,9)$ & $98(18,6)$ & $227(12,8)$ \\
\hline Evaluar & $16(5,1)$ & $35(7,2)$ & $64(14,1)$ & $61(11,6)$ & $176(9,9)$ \\
\hline Crear & $10(3,2)$ & $14(2,9)$ & $24(5,3)$ & $15(2,8)$ & $63(3,5)$ \\
\hline Total & $311(100)$ & $483(100)$ & $453(100)$ & $528(100)$ & $1775(100)$ \\
\hline
\end{tabular}

Fuente: Elaboración propia (2020) 
La dimensión de los procesos cognitivos es una clasificación de dominios. Se organizan en función de un continuo de complejidad cognitiva, las que asumen que el aprendizaje en niveles superiores depende de la adquisición del conocimiento y las habilidades de ciertos niveles inferiores (MIDE, 2013), hecho que permitió aplicar a los datos, los test estadístico no paramétricos $H$ Kruskal-Wallis, Chi cuadrado y Rho de Spearman).

En la Tabla 3 se constatan diferencias estadísticamente significativas entre las dimensiones del proceso cognitivo de las preguntas formuladas en los textos de Historia, Geografía y Ciencias Sociales y el curso en el cual se plantean ( $H$ Kruskal-Wallis, $p=0,000)$.

Tabla 3

Resumen de prueba de hipótesis

\begin{tabular}{lllll}
\hline & Hipótesis nula & Prueba & Sig. & Decisión \\
\hline 1 & $\begin{array}{l}\text { La distribución de procesos } \\
\text { cognitivos es la misma entre las } \\
\text { categorías de curso }\end{array}$ & $\begin{array}{l}\text { Prueba de Kruskal-Wallis } \\
\text { para muestras } \\
\text { independientes }\end{array}$ & & $\begin{array}{l}\text { Rechazar la } \\
\text { hipótesis nula }\end{array}$ \\
\hline
\end{tabular}

Fuente: Elaboración propia (2020)

El test mostró la existencia de diferencias estadísticamente significativas en al menos una pareja de cursos de seis posibles combinaciones entre la dimensión del proceso cognitivo de las preguntas formuladas en los textos escolares de Historia, Geografía y Ciencias Sociales de este estudio. Se da cuenta de la existencia de diferencias estadísticamente significativas entre 4 parejas de cursos, a saber: $5 .^{\circ}-8 .^{\circ}, 5 .^{\circ}-7 .^{\circ}, 6 .^{\circ}-8 .^{\circ}$ y $6 .^{\circ}-7 .^{\circ}(U$ de Mann-Whitney, $p=0,000)$. También, el test consigna que dos parejas de cursos no presentan diferencias estadísticamente significativas, esto es, $5 .^{\circ}-6 .^{\circ}$ y $7 .^{\circ}-8 .^{\circ}(U$ de Mann-Whitney, $p=1,000)$. Los valores se muestran en la Tabla 4.

Tabla 4

Nivel de significancia entre 6 parejas de cursos ( $U$ de Mann-Whitney)

\begin{tabular}{llllll}
\hline $\begin{array}{l}\text { Muestra 1- } \\
\text { Muestra 2 }\end{array}$ & $\begin{array}{l}\text { Estadístico de } \\
\text { contraste }\end{array}$ & $\begin{array}{l}\text { Error } \\
\text { estándar }\end{array}$ & $\begin{array}{l}\text { Desv. estadístico } \\
\text { de contraste }\end{array}$ & Sig. & $\begin{array}{l}\text { Sig. } \\
\text { ajust. }\end{array}$ \\
\hline $5 .^{\circ}-6 .^{\circ}$ & $-34,874$ & 35,412 &,- 985 &, 325 & 1,000 \\
$5 .^{\circ}-8 .^{\circ}$ & $-180,017$ & 34,816 & $-5,171$ &, 000 &, 000 \\
$5 .^{\circ}-7 .^{\circ}$ & $-194,984$ & 35,869 & $-5,436$ &, 000 &, 000 \\
$6 .^{\circ}-8 .^{\circ}$ & $-145,143$ & 30,668 & $-4,733$ &, 000 &, 000 \\
$6 .^{\circ}-7 .^{\circ}$ & $-160,110$ & 31,858 & $-5,026$ &, 000 &, 000 \\
$8 .^{\circ}-7 .^{\circ}$ & 14,966 & 31,194 &, 480 &, 631 & 1,000 \\
\hline
\end{tabular}

Fuente: Elaboración propia (2020) 
Para determinar la existencia de una asociación entre las dimensiones del proceso cognitivo de las preguntas y el curso en el cual se plantean, se aplicó el test Rho de Spearman $(0,00$ 0,19=muy baja; 0,20-0,39=baja; 0,40-0,59=moderada; 0,60-0,79=buena; 0,80-1,00=muy buena). Este constata que existe asociación con un 0,01 de significancia, sin embargo, esta asociación es muy baja con un $r_{s}=0,153$ (Tabla 5).

Tabla 5

Potencia de correlación entre procesos cognitivos de las preguntas y curso, asociados al coeficiente Rho de Spearman

\begin{tabular}{llcc}
\hline & & Proceso cognitivo & Curso \\
\hline Proceso cognitivo & Coef. de correlación & 1,000 &, 153 \\
& Sig. (bilateral) & $\cdot$ &, 000 \\
& $\mathrm{~N}$ & 1775 & 1775 \\
Curso & Coef. de correlación &, 153 & 1,000 \\
& Sig. (bilateral) &, 000 & $\cdot$ \\
& $\mathrm{N}$ & 1775 & 1775 \\
\hline
\end{tabular}

Fuente: Elaboración propia (2020)

\section{Dimensión del proceso cognitivo de las preguntas y unidades temáticas.}

En la Tabla 6 se da cuenta de la dimensión cognitiva que movilizan las preguntas de acuerdo con las unidades temáticas. Se advierte que todas las unidades promueven, mayoritariamente, la dimensión recordar, disminuyendo su valor al ser más alta la dimensión cognitiva de la taxonomía. Cabe relevar que no se observan diferencias significativas en las dimensiones del proceso cognitivo de las preguntas entre las unidades temáticas de los textos escolares estudiados.

Tabla 6

Frecuencia (y porcentaje) del proceso cognitivo, por unidad temática, de las preguntas escritas

\begin{tabular}{lllllll}
\hline Proceso cognitivo & Unidad 1 & Unidad 2 & Unidad 3 & Unidad 4 & Unidad 5 & Total \\
\hline Recordar & $137(41)$ & $201(46,2)$ & $204(43,9)$ & $136(43,9)$ & $91(39,4)$ & $769(43,3)$ \\
Comprender & $76(22,8)$ & $100(23)$ & $101(21,7)$ & $76(24,5)$ & $54(23,4)$ & $407(22,9)$ \\
Aplicar & $22(6,6)$ & $35(8)$ & $40(8,6)$ & $19(6,1)$ & $17(7,4)$ & $133(7,5)$ \\
Analizar & $47(14,1)$ & $55(12,6)$ & $60(12,9)$ & $41(13,2)$ & $24(10,4)$ & $227(12,8)$ \\
Evaluar & $40(12)$ & $33(7,6)$ & $46(9,9)$ & $26(8,4)$ & $31(13,4)$ & $176(9,9)$ \\
Crear & $12(3,6)$ & $11(2,5)$ & $14(3)$ & $12(3,9)$ & $14(6,1)$ & $63(3,5)$ \\
\hline Total & $334(100)$ & $435(100)$ & $465(100)$ & $310(100)$ & $231(100)$ & $1775(100)$ \\
\hline
\end{tabular}

Fuente: Elaboración propia (2020) 
Cabe preguntarse: ¿Las dimensiones del proceso cognitivo de las preguntas formuladas en los textos escolares son estadísticamente diferentes según la unidad en la cual se plantean? Se aplicó el estadístico $H$ de Kruskal-Wallis. Se asume el valor 7,22 que, con 4 grados de libertad, un $p$-valor $=0,13$ y un nivel de significancia del 5\%, indica que no existen diferencias estadísticamente significativas en las dimensiones del nivel cognitivo de las preguntas de los textos, según la unidad temática en la cual se plantean.

Los resultados de la aplicación del Chi-cuadrado muestra que no existe asociación entre el proceso cognitivo de las preguntas que se formulan en la muestra de los textos de Historia, Geografía y Ciencias Sociales y la unidad en la que se plantean, $\chi^{2}(20, \mathrm{~N}=1.775)=19,65, p=$ 0,48 " (Tabla 7), que es equivalente a mencionar que el proceso cognitivo de las preguntas que se formulan no tiene ninguna relación con la unidad en la cual se plantean.

Tabla 7

Pruebas de Chi-cuadrado y resultados

\begin{tabular}{lccc}
\hline & Valor & Df & $\begin{array}{c}\text { Significación } \\
\text { asintótica (bilateral) }\end{array}$ \\
\hline Chi-cuadrado de Pearson & 19,649 & 20 &, 480 \\
Razón de verosimilitud & 19,059 & 20 &, 518 \\
Asociación lineal por lineal &, 654 & 1 &, 419 \\
N de casos válidos & 1775 & & \\
\hline
\end{tabular}

Fuente: Elaboración propia (2020)

\section{Discusión de resultados}

De acuerdo con los resultados de esta investigación, se puede señalar que existen coincidencias con otros estudios. Campbell y Mayer (2009) señalan que las preguntas adjuntas a textos favorecen procesos cognitivos de selección, organización e integración de información, lo cual posibilita aprendizajes de calidad.

Hamaker (1986) también encontró que las preguntas de alto nivel de cognición mejoran la comprensión, a diferencia de las preguntas simples, que son factuales y promueven el aprendizaje memorístico. Sin embargo, en este estudio, se constata que las preguntas de comprensión fluctúan entre el 25 y 21,5\% en los cuatro cursos. Es decir, en los textos se promueven bajas dimensiones 
del proceso cognitivo. Asimismo, en investigaciones de Wimer et al. (2001) se reafirma que las preguntas de alto nivel promueven habilidades de análisis, síntesis y evaluación. Esto incide en el desarrollo del pensamiento crítico, en cambio, las preguntas de bajo nivel demandan solo habilidades de recuerdo y memoria.

Por otra parte, en relación con preguntas formuladas en textos expositivos y narrativos, Graesser (1981) sostuvo que, en textos narrativos, los lectores podían realizar cuatro veces más inferencias que en los expositivos. Esto es importante de señalar, ya que las preguntas de los textos escolares de Historia, Geografía y Ciencias Sociales de educación básica chilena estarían asociadas a textos expositivos. Esto incide a su vez en la calidad y cantidad de inferencias que puedan realizar los alumnos de este nivel y, por ende, impacta en el desarrollo del pensamiento histórico y en los tipos de aprendizajes.

Valls (2007) señala que los textos escolares del siglo XIX presentaban los contenidos mediante "una forma catequística de preguntas y respuestas" (p. 76), que facilitaba la memorización, lo cual no difiere mucho de los textos escolares analizados en esta investigación. De la misma forma, Mahamud y Badanelli (2016) señalan que la estructura de los textos escolares requiere de un "lector modelo" que responde a un estereotipo de sujeto que lee, memoriza o copia los textos presentados. Se evidencia que los textos no fomentan el desarrollo de habilidades cognitivas superiores, en sus diversas secciones y, por ende, no se puede esperar el fomento del pensamiento histórico, el cual requiere movilizar una serie de capacidades intelectuales. Las preguntas contenidas en las actividades didácticas deberían promover el desarrollo de este tipo de habilidades.

De acuerdo con las taxonomías de Trabasso y Magliano (1996) e Ishiwa, San José y Otero (2013) que establecen preguntas de tipo: asociativas, explicativas y predictivas, se puede señalar, que en esta investigación se encontraron, mayoritariamente, preguntas asociativas buscando responder qué, cómo, cuándo y dónde. En menor medida se identificaron preguntas que demandaban respuestas explicativas (por qué) y, la menor proporción de preguntas estuvo centrada en respuestas que requerían inferencias predictivas (qué pasaría si...)

Sáiz y Colomer (2014) categorizaron las actividades didácticas contenidas en textos escolares de acuerdo con la capacidad cognitiva en tres niveles de complejidad y concluyeron que la menor cantidad de actividades didácticas se concentra en el nivel más complejo. En este estudio se encontraron resultados similares en el análisis de las preguntas contenidas en los textos escolares de Historia, Geografía y Ciencias Sociales de la enseñanza básica chilena. Por otra parte, Gómez 
y Miralles (2013) refrendan el peso abrumador de las preguntas de baja dimensión cognitiva, de reproducción de contenidos factuales en los textos escolares.

En síntesis, trabajos relacionados con la formulación de preguntas coinciden con los hallazgos de esta investigación en los niveles de razonamiento y dimensiones del proceso cognitivo. Es decir, las preguntas formuladas en los textos escolares se encuentran en los niveles y dimensiones inferiores, por lo que se puede colegir que no hay una intención para desarrollar pensamientos más complejos en los estudiantes mediante los textos escolares.

\section{Conclusiones}

Se ha detectado que las preguntas clasificadas en la dimensión recordar se encuentran entre $53,1 \%$ a $36,4 \%$ en los textos de $5 .^{\circ}$ a $8 .^{\circ}$ año de enseñanza básica chilena. Existe una mayor concentración de preguntas en esta dimensión. Preguntas más complejas, clasificadas en las dimensiones superiores de la taxonomía, se presentan solo en el último curso ( $8 .^{\circ}$ año).

El nivel superior de la taxonomía concentra el 33,7 \%, que se desglosa por dimensión del proceso cognitivo en la siguiente proporción: 7,5\% en aplicar; $12,8 \%$ en analizar; 9,9 \% en evaluar y 3,5\% en crear. La dimensión intermedia correspondiente al comprender fluctúa entre el 25 y $21,5 \%$ de las preguntas en los cuatro cursos, de $5 .^{\circ}$ a $8 .^{\circ}$ año básico.

En cuanto al análisis del tipo de dimensión cognitiva de las preguntas, se comprueba que el texto escolar fomenta, sobretodo, preguntas de nivel memorístico y reproductivo. Estas últimas, son porcentualmente mayores que las preguntas que hacen referencia al nivel cognitivo superior, y por tanto, un menor desarrollo de competencias intelectuales. Este tipo de preguntas es, pese a toda su importancia, tratado en un porcentaje minoritario. Los procesos cognitivos de las preguntas formuladas en los textos escolares analizados difieren entre los niveles $5 .^{\circ}-6 .^{\circ} \operatorname{con} 7 .^{\circ}-8 .^{\circ}$ no así, entre los subniveles: $5 .^{\circ} \operatorname{con} 6 .^{\circ}$ y $7 .^{\circ} \operatorname{con} 8 .^{\circ}$ año de enseñanza básica.

Los resultados obtenidos evidencian la necesidad de incorporar, en mayor proporción, preguntas de dimensiones cognitivas superiores, y también, establecer diferencias entre las dimensiones cognitivas de las preguntas destinadas a los diferentes niveles educativos de educación básica. Se constata la presencia de cuatro "parejas de cursos" en los que difieren el nivel taxonómico de las preguntas formuladas. Los resultados obtenidos permiten concluir que las dimensiones cognitivas subyacentes en las preguntas promovidas en los textos escolares, no 
reportan una contribución clara y suficiente para generar preguntas que movilicen un desarrollo intelectual superior en los estudiantes.

Se encontró que existen “duplas de cursos” y diferencias en los grados de complejidad de las preguntas. Además, se pudo constatar ausencia de asociación entre ellos, es decir, no existe una correspondencia entre unidades y dimensión cognitiva superior en las preguntas formuladas en los textos. Esperable hubiese sido encontrar una relación directamente proporcional entre el aumento de complejidad de las preguntas y el desarrollo de las unidades a lo largo del texto escolar. Sin embargo, esto no ocurre.

El análisis de la dimensión del proceso cognitivo de las preguntas formuladas en los textos escolares, según el curso en el cual se plantean, indica que existen diferencias estadísticamente significativas en cuatro parejas de cursos, esto es, $5 .^{\circ}-8 .^{\circ}, 5 .^{\circ}-7 .^{\circ}, 6 .^{\circ}-8 .^{\circ}$ y $6 .^{\circ}-7 .^{\circ}$ básico. Sin embargo, entre las parejas de cursos $5 .^{\circ}-6 .^{\circ}$ y $7 .^{\circ}-8 .^{\circ}$ no se observan diferencias significativas. Tampoco existen diferencias en la dimensión del proceso cognitivo de las preguntas y las unidades temáticas en los textos analizados. Si bien es cierto que se encontró, estadísticamente, la existencia de asociación entre las dimensiones del proceso cognitivo de las preguntas y el curso en el cual se plantean, el valor es cercano a cero, lo cual indica ausencia de asociación entre variables.

Finalmente, se debe señalar, que las preguntas contenidas en los textos escolares de Historia, Geografía y Ciencias Sociales de la enseñanza básica chilena se promueve, escasamente, el desarrollo del pensamiento histórico, a pesar de evidencias acerca de la posibilidad de fomentarlo desde temprana edad.

El desafío es incorporar preguntas a las actividades didácticas de los textos escolares para desarrollar, de forma progresiva, las habilidades cognitivas relacionadas con el pensamiento histórico.

\section{Referencias}

Anderson, L. W., y Krathwohl, D. (2001). A Taxonomy for learning, teaching and assessing: a revision of Bloom's taxonomy of educational objectives. New York, NY: Longman.

André, T. (1990). Type of inserted questions and the study post-test delay. Journal of experimental Education, 58(2), 77-86.

Bel, J. C., y Colomer, J. C. (2018). Teoría y metodología de investigación sobre libros de texto: análisis didáctico de las actividades, las imágenes y los recursos digitales en la enseñanza de las Ciencias Sociales. Revista Brasileira de Educação, 23, e230082. 
Berlyne, D. E. y Frommer, F. D. (1966). Some determinants of the incidence and content of children's questions. Child Development, 37(1), 177-189.

Boudreau, R. L., Wood, E., Willoughby, T. y Specht, J. (1999). Evaluating the efficacy of elaborative strategies for remembering expository text. Alberta Journal of Educational Research, 45(2), 170-183.

Campbell, J., y Mayer, R. E. (2009). Questioning as an instructional method: does it affect learning from lectures? Applied Cognitive Psychology, 23(6), 747-759.

Carretero, M., y Montanero, M. (2008). Enseñanza y aprendizaje de la Historia: aspectos cognitivos y culturales. Cultura y Educación, 20(2), 133-142

Dillon, J. T. (1990). The practice of questioning. Nueva York, NY: Routledge.

Escobar-Pérez, J., y Cuervo-Martínez, A. (2008). Validez de contenido y juicio de expertos: una aproximación a su utilización. Avances en Medición, 6(1), 27-36.

Fernández, C. y Giadronic, G. (2018a). Historia, Geografía y Ciencias Sociales 5 Básico. Santiago, Chile: SM.

Fernández, C. y Giadronic, G. (2018b). Historia, Geografía y Ciencias Sociales 6º Básico. Santiago, Chile: SM.

Flammer, A. (1981). Towards a theory of question asking. Psychological Research, 43(4), 407-420.

Friedman, F., y Rickards, J. P. (1981). Effect of level, review, and sequence of inserted questions on text processing. Journal of Educational Psychology, 73(3), 427-436.

Gallástegui, J. R., y Otero, J. (2008). Podemos avaliar a calidade das preguntas do alumnado? Boletin das Ciencias, 66, 195-197.

Gavelek, J. R., y Raphael, T. E. (1985). Metacognition, instruction, and the role of questioning activities. En D. L. Forrest-Pressley, G. E. Mackinnon, T. G. Waller (Eds.), Metacognition, cognition and human performance (pp. 103-136). Orlando, FL: Academic Press.

Gómez, C. J., y Miralles, P. (2013). Los contenidos de Ciencias Sociales y las capacidades cognitivas en los exámenes de tercer ciclo de educación Primaria ¿Una evaluación de competencias? Revista Complutense de Educación, 24(1), 91-121.

Graesser, A. C. (1981). Prose comprehension beyond the world. Nueva York, NY: Springer-Verlag.

Graesser, A. C., y Olde, B. (2003). How does one know whether a person understands a device? The quality of the questions the person asks when the device breaks down. Journal of Educational Psychology, 95(3), 524-536.

Graesser, A. C., McMahen, C. L., y Johnson, B. K. (1994). Question asking and answering. En M. A. Gernbascher (Ed.), Handbook of Psycholinguistics (pp. 517-538). New York, NY: Academic Press

Hamaker, C. (1986). The effects of adjunct questions on prose learning. Review of Educational Research, 56(2), 212242.

Ishiwa, K., San José, V. y Otero, J. (2013). Generation of information-seeking questions on scientific texts under different reading goals. British Journal of Education Psychology, 83(3), 502-520.

King, A. (1995). Inquiring minds really do want to know: using questioning to teach critical thinking. Teaching of Psychology, 22(1), 13-17.

La France, M. (1992). Questioning knowledge acquisition. En T. Lauer, E. Peacock y A. C. Graesser (Eds.), Questions and information systems (pp. 11-28). Hillsdale, NJ: Erlbaum.

Landa, L. y Pinto, V. (2018a). Historia y Geografía y Ciencias Sociales $7^{\circ}$ Básico. Santiago, Chile: SM.

Landa, L. y Pinto, V. (2018b). Historia y Geografía y Ciencias Sociales $8^{\circ}$ Básico. Santiago, Chile: SM. 
Mahamud, K., y Badanelli, A. M. (2013). El cuaderno escolar como objeto de estudio: una aproximación a los avances metodológicos en manualística. En J. Meda y A. Badanelli (Eds.), La historia de la cultura escolar en Italia y España: balances y perspectivas (pp. 201-228). Macerata: Edizione Universitá di Macerata.

McDaniel, M. A., y Donnelly, C. M. (1996). Learning with analogy and elaborative interrogation. Journal of Educational Psychology, 88(3), 508-519.

MIDE. (2013). Construcción de ítems taxonomía de habilidades cognitivas. Santiago, Chile: Universidad Católica de Chile.

Otero, J., y Graesser, A. C. (2001). PREG: Elements of a model of question asking. Cognition and Instruction, 19(2), $143-175$.

Ozgungor, S., y Guthrie, J. T. (2004). Interactions among elaborative interrogation, knowledge, and interest in the process of constructing knowledge from text. Journal of Educational Psychology, 96(3), 437-443.

Pressley, M., McDaniel, M. A., Turnure, J. E., Wood, E., y Ahmad, M. (1987). Generation and precision of elaboration: Effects on intentional and incidental learning. Journal of Experimental Psychology: Learning, Memory, and Cognition, 13(2), 291-300.

Pressley, M., Symons, S., McDaniel, M., Snyder, B., y Turnure, J. (1988). Elaborative interrogation facilitates the acquisition of confusing facts. Journal of Educational Psychology, 80(3), 268-278.

Ram, A. (1991). A theory of questions and question asking. The Journal of the Learnig Scienciences, 1(3-4), 273-318.

Sáez-Rosenkranz, I. (2017). La enseñanza de la Historia en los libros de texto de educación básica en Chile. Enseñanza de las Ciencias Sociales, 16, 27-40.

Sáiz, J., y Colomer, J. C. (2014). ¿Se enseña pensamiento histórico en libros de texto de Educación Primaria? CLIO. History and History Teaching, 40, 1-19.

Seifert, T. L. (1993). Effects of elaborative interrogation with prose passages. Journal of Educational Psychology, $85(4), 642-651$.

Torres, T., Duque, J., Ishiwa, K., Sánchez, G., Solaz Portolés, J. J., y San José, V. (2012). Preguntas de los estudiantes de educación secundaria ante dispositivos experimentales. Enseñanza de las Ciencias, 30(1), 49-60.

Trabasso, T., y Magliano, J. P. (1996). Conscious understanding during text comprehension. Discourse Processes, 21(2), 255-287.

Valls, R. (2007). Historiografía Escolar Española Siglos XIX-XXI. Madrid, España: UNED.

Van den Broek, P.W., Tzeng, Y., Risden, K., Trabasso, T., y Basche, P. (2001). Inferential questioning: Effects on comprehension of narrative texts as a function of grade and timing. Journal of Educational Psychology, 93(3), 521-529.

Van Der Meij, H. (1990). Question asking: to know that you do not know is not enough. Journal of Educational Psychology, 82(3), 505-512.

Van Der Meij, H. (1994). Student questioning: a componential analysis. Learning and Individual Differences, 6(2), 137-171.

Wimer, J. W., Ridenour, C. S., Thomas, K., y Place A. W. (2001). Higher order teacher questioning of boys and girls in elementary mathematics classrooms. The Journal of Educational Research, 95(2), 84-92.

Woloshyn, V. E., Wood, E., Willoughby, T., y Pressley, M. (1990). Elaborative interrogation facilitates adult learning of factual paragraphs. Journal of Educational Psychology, 82(3), 513-524. 
Wood, D. (1988). How Children Think and Learn. Oxford: Basil Blackwell.

Wood, E., Pressley, M., y Winne, P. (1990). Elaborative interrogation effects on children's learning of factual content. Journal of Educational Psychology, 82(4), 741-748.

\section{Contribución autoral}

a) Concepción y diseño del trabajo; b) Adquisición de datos; c) Análisis e interpretación de datos; d) Redacción del manuscrito; e) revisión crítica del manuscrito.

A. M. M. ha contribuido en a, d, e; A. S. O. en a, b, c; D. D-L. en b, e; D. I. O. en c.

\section{Editora científica responsable}

Mag. Florencia de León 